\title{
Computational Model for Calculation Horizontal Forces on Crane Runway
}

\author{
Josef Musilek ${ }^{1, *}$ \\ ${ }^{1}$ Institute of Technology and Business in České Budějovice, Department of Civil Engineering, \\ Okružní 517/10, 37001 České Budějovice, Czech Republic
}

\begin{abstract}
During a motion of an overhead bridge crane horizontal forces between wheels of the crane and the crane runway occur. These forces can be caused by skewing, acceleration or braking of the crane. There are several procedures for determination of these forces. These procedures can be found in professional literature and also in present standards for check and designing of crane structures. However, when there is made a comparison of computational models presented in the literature and present standards, it turns out that the computational models are different even if they describe the same physical situation - for example skewing of the crane or the acceleration or braking of the crane. The aim of this paper is to create computational model which would describe real behaviour of the crane during its motion on the crane runway and which would also enable to calculate the horizontal transverse forces between the crane wheels and the crane runway. This model will be created by modifying of the model already created by another author by taking account influences affecting the crane motion on the crane runway.
\end{abstract}

\section{Introduction}

There are several procedures and models for calculation of horizontal forces acting on the crane runway during motion of the overhead bridge crane. The choice of the model and procedure depends of the kind of horizontal forces which is needed to calculate. But if the comparison of the models and procedures is made and the results got from these different models are compared, the different magnitudes of horizontal forces are obtained. This is shown for example in [1] where there are compared computational models according [2-4]. In the following text there are described two models which approach the determining of horizontal forces as a dynamic task. These dynamic models try to include substantial influences which can influence the motion of the crane and therefore also the horizontal forces between the crane and the crane runway.

\footnotetext{
* Corresponding author: musilek@mail.vstecb.cz
} 


\section{Dynamic model according to Lobov}

Lobov $[5,6]$ solve the determination horizontal forces as a dynamic task. The result of the solution is the dynamic model with kinematic variables describing the crane movement along the crane path and also the size of the lateral forces between the crane wheels and the crane track. In the models he assembled, Lobov solved two crane movement situations: the first of them without contact the guide means with the crane path; in the second situation, he considered touching the guide means with the side of the rails. The model designed by Lobov allows to adjust the position of the crane crab to the desired position, which changes the weight distribution after the crane design. When defining the drive forces on a crane, it is based on torque characteristics of the motors, which are considered to be straightforward, so that the behavior of the crane with motors with different characteristics can be simulated. The Lobov model also introduced the possibility of sloping crane wheels with respect to the crane cross-axis, which may be due to manufacturing imperfections.

Lobov also considered the crane's flexibility by introducing a flexible crane wheel support on the end carriages, which involves the deformation of the end carriage during motion. On the contrary, in his models he neglected the rigidity of the crane bridges and considered them a perfectly stiff body and did not consider the influence of the load swinging. When modeling the drive, the crane also did not consider the drive regulation currently used to drive bridge cranes.

Fig. 1 shows the crane model according to Lobov in case the guide means do not touch the rails.

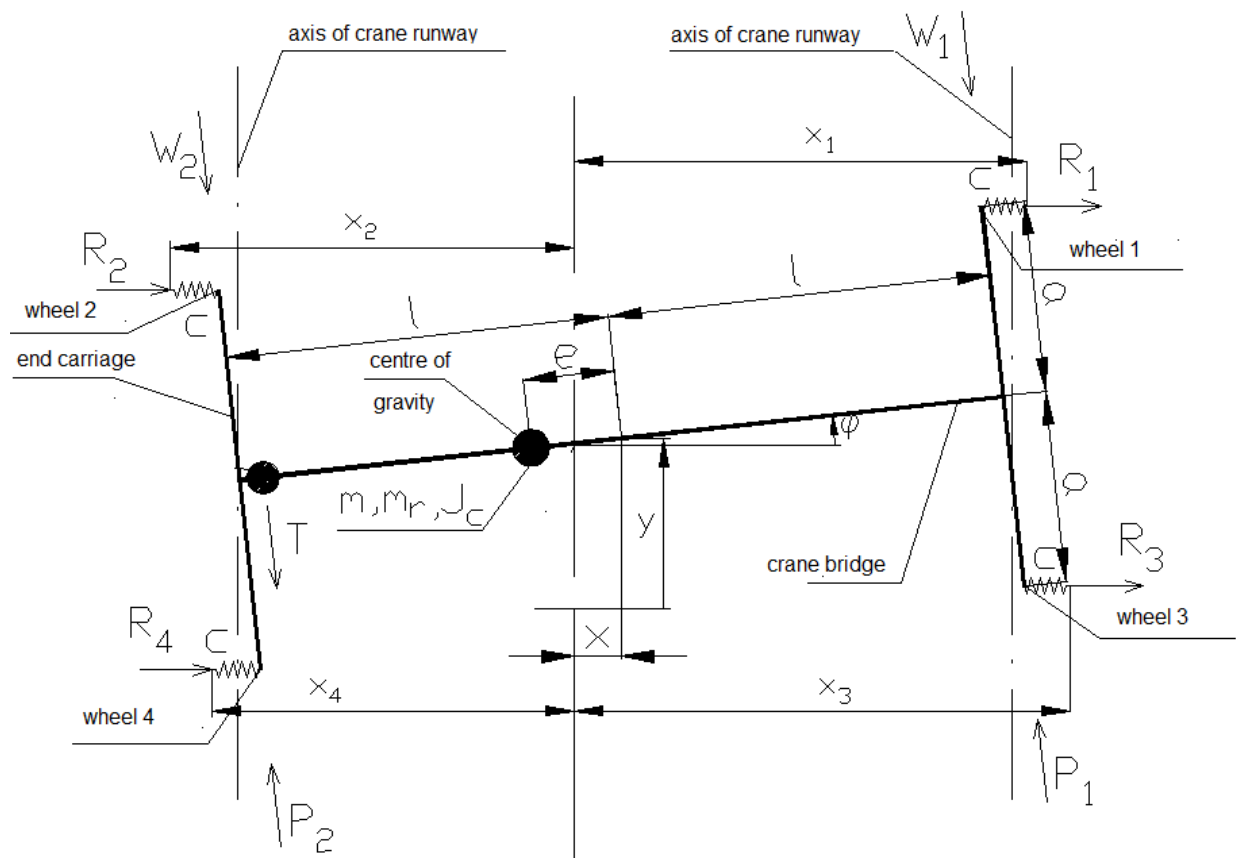

Fig. 1 Dynamic model

In Fig. 1 are:

mass of the crane reduced to the longitudinal direction of the crane (in y direction); includes the weight of the crane crane, the rotating 
mass of the crane drive, and is used to write the motion equations in $-\mathrm{m}, \mathrm{Jc}$ the direction of the $y$

crane to the center of gravity of the crane; they include the weight of
the crane with the cat and are used to write the motion equations in
the direction of the searched coordinate $\mathrm{x}$ and $\varphi$
bending stiffness of the free ends of the end carriages
- c
the transverse force between the crane wheels and the crane track
the resistance forces generated by the rolling of the wheel and
- W1, W2
always acting against the movement of the crane; are considered
constant during crane driving
the driving forces of the motors
-P1, P2
horizontal force in the lifting rope of the crab, arising from the
swinging of the load

The transverse forces R1, R2, R3, R4 are the forces that load the structure of the crane runway and the construction of the crane. They are determined on the basis of the linear slip. This is described by the following equation:

$$
R=-K \cdot \sigma_{y}
$$

where:

$-\mathrm{K}$

$-\sigma_{\mathrm{y}}$

is the slip constant

is the crane wheel transverse sliding; this slip is based on the analysis of kinematic conditions

Determining the slip constant $\mathrm{K}$ is a relatively challenging problem which is solved by contact mechanics theory. Various relations for the calculation of the slip constant of $\mathrm{K}$ are presented. Lobov presents the following formula for K:

$$
K=5,344 \cdot 10^{4} \cdot \sqrt{R_{k} \cdot G}
$$

where:

- $\mathrm{R}_{\mathrm{k}} \quad$ is radius of crane wheel [m]

- $\mathrm{G} \quad$ is wheel load of crane wheel [N]

For construction of motion equations are used Lagrange equations. Kinetic energy of the system has the following form:

$$
E_{0}=\frac{1}{2} \cdot m_{r} \cdot(\dot{y}-e \cdot \dot{\varphi})^{2}+\frac{1}{2} \cdot m \cdot \dot{x}^{2}+\frac{1}{2} \cdot J_{c} \cdot \dot{\varphi}^{2}
$$

After substitution the kinetic energy into the Lagrange equations and expressing the generalized forces by the principle of equality of instantaneous powers, we get the motion equations of the system:

$$
\begin{gathered}
m_{r} \cdot(\ddot{y}-e \cdot \ddot{\varphi})=P_{1}+P_{2}-W_{1}-W_{2}-T \\
m \cdot \ddot{x}=R_{1}+R_{2}+R_{3}+R_{4} \\
\left(J_{c}+m \cdot e^{2}\right) \cdot \ddot{\varphi}-m \cdot e \cdot \ddot{y}=\left(P_{1}-P_{2}+W_{2}-W_{1}+T\right) \cdot l-\left(R_{1}+R_{2}-R_{3}-R_{4}\right) \cdot a
\end{gathered}
$$

In the following there is considered $\mathrm{T}=0$. It is also assumed that the crane is already in a uniform motion with constant velocity. Then, P1 $+\mathrm{P} 2+\mathrm{W} 1+\mathrm{W} 2=0$ and. The system of equations is thus simplified to the form where $\mathrm{x}, \varphi$ are unknown. After solving the system of motion equations we get the course $\mathrm{x}$ and $\varphi$ at a time, by which we gradually calculate 
the transverse slides on the individual wheels. By placing it in relation (1) we obtain the desired transverse forces $\mathrm{R}$ on the individual wheels.

$$
\begin{gathered}
m \cdot \ddot{x}=R_{1}+R_{2}+R_{3}+R_{4} \\
\left(J_{c}+m \cdot e^{2}\right) \cdot \ddot{\varphi}=-\left(R_{1}+R_{2}-R_{3}-R_{4}\right) \cdot a
\end{gathered}
$$

\section{Modified dynamic model}

The dynamic model presented above doesn't include influence of the load swinging. The load swinging occurs very often during motion of the overhead bridge crane and can have impact on the motion of the crane. This fact can be taken into account by following modification. The modified model can be seen on

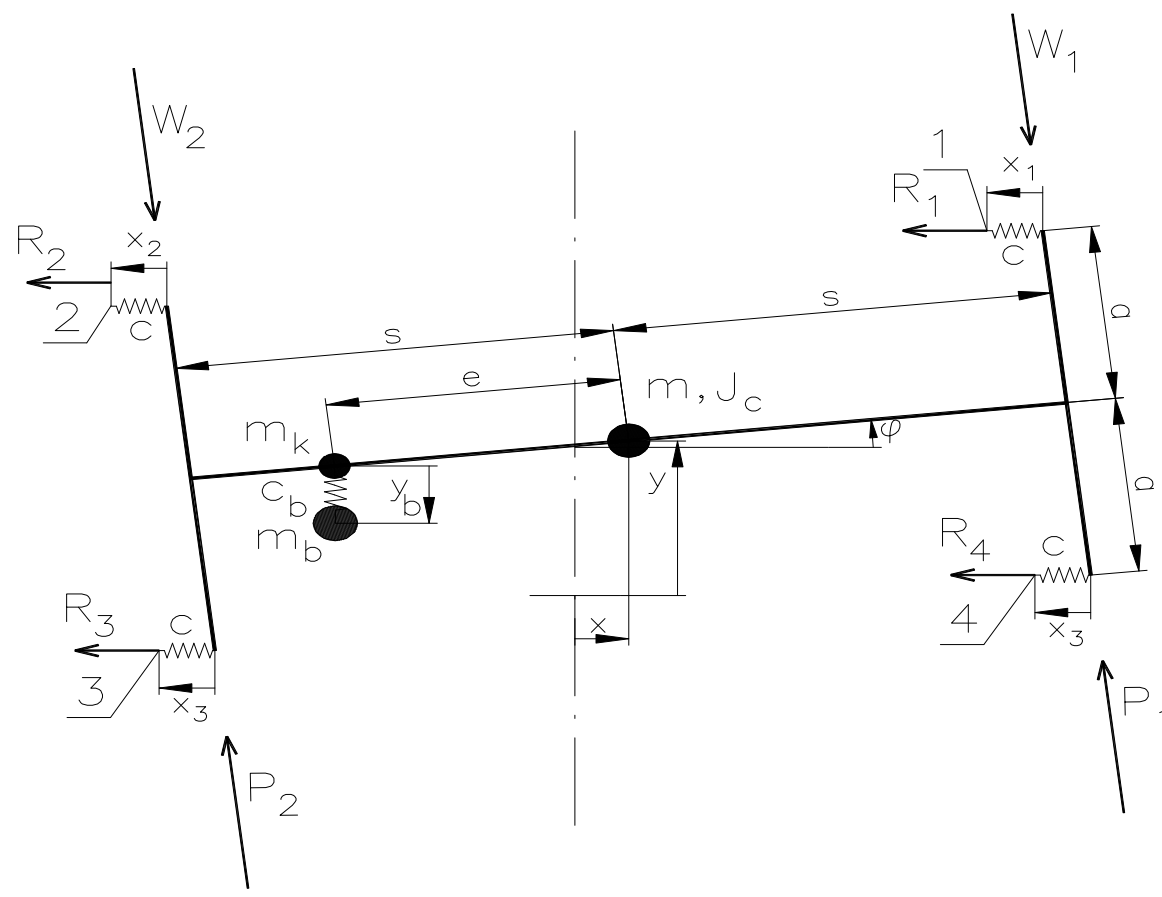

Fig. 2 Modified dynamic model

The model was modified mainly by adding the mass of the crab. The mass of the crab was removed from the mass of the crane. The mass of the crab was put on its real position which is taken into account for calculation.

Then the mass of the load was added. The mass of load is connected to the mass of the crab by spring. The stiffness of this spring simulates the interaction between swinging load and the crane when the crane is moving and the load is swinging.

Let's assume relatively small deflection of load during the motion of the crane. Than the value of the spring stiffness can be derived from the similarity between mathematical pendulum and the oscillation of the mass on the spring. If we compare the equations of motions of mathematical pendulum and the oscillation of the mass on the spring, we get the following formula: 


$$
c_{b}=\frac{m \cdot g}{l}
$$

where:

$-\mathrm{m}_{\mathrm{k}} \quad$ is mass of the load $[\mathrm{kg}]$

$-1 \quad$ is length of the rope [N]

\section{Conclusions}

Two models for determination of horizontal forces acting on crane runway during motion of an overhead bridge crane were presented in the paper. These both model are dynamical and try to respect the physical principle of the crane driving on the crane runway by inclusion of the influences which have the impact on the motion of the crane and hence the magnitude of the horizontal forces. The second model was created by modification of the first one. The real position of the crab mass was added while in the first mode the mass of the crab was taken as the part of the crane. The mass of the load was also added in the second model. The swinging of the load on the rope during the motion of the crane was taken into account. The load swinging of the load was taken into account by using of a spring between the crab and load. The reason for this modification is to prepare a dynamic model which is more about real behaviour.

\section{References}

1. J. Musilek, World Multidisciplinary Civil Engineering-Architecture-Urban Planning Symposium 2018 (to be published)

2. EN 1991-3. Eurocode 1 - Actions on structures - Part 3: Actions induced by cranes and machinery (2006)

3. ČSN 73 0035. Action on structure of buildings (ÚNM Prague, 1986)

4. ČSN 27 0103. Design of steel structures of cranes (ÚNM Prague, 1989)

5. N.A. Lobov, Bulletin of Machine Building, 62, 31-35 (1982)

6. N.A. Lobov, Bulletin of Machine Building, 64, 22-26 (1984) 